\title{
Hepatic Arterial Infusion Chemotherapy Is a Feasible Treatment Option for Hepatocellular Carcinoma: A New Update
}

\author{
Maher Hendi ${ }^{a}$ Yiping Mou ${ }^{b} \quad$ Jiemin Lva Bin Zhang $^{a} \quad$ Xiujun Cai ${ }^{a}$ \\ aDepartment of Surgery, Sir Run-Run Shaw Hospital, Zhejiang University School of Medicine, Hangzhou, China; \\ ${ }^{b}$ Department of Surgery, Zhejiang Provincial Peoples Hospital, Hangzhou, China
}

\section{Keywords}

Hepatocellular carcinoma $\cdot$ Hepatic artery infusion

chemotherapy · Transarterial chemoembolization · FOLFOX

\section{Abstract}

Background: Hepatic arterial infusion chemotherapy (HAIC) is one option for treating massive tumors and unresectable hepatocellular carcinoma (HCC). However, there is a lack of remedial treatment after these treatments are ineffective or failed. Summary: Some studies have discovered that HAIC has greater survival in patients with advanced HCC. A previous study has shown that HAIC is effective in the treatment of advanced HCC, and the data on randomized clinical trials are limited and unclear. Key Message: More clinical trials and research are needed in order to make HAIC a standard and recommended therapy for advanced HCC. Our review focuses on the clinical applications of hepatic artery infusion treatment.

(C) 2021 The Author(s).

Published by S. Karger AG, Basel

karger@karger.com www.karger.com/gat

Karger"

BOPEN ACCESS
(C) 2021 The Author(s)

Published by S. Karger AG, Basel

This is an Open Access article licensed under the Creative Commons Attribution-NonCommercial-4.0 International License (CC BY-NC) (http://www.karger.com/Services/OpenAccessLicense), applicable to the online version of the article only. Usage and distribution for commercial purposes requires written permission.

\section{Introduction}

Regardless of gender or country, there is an increase in the number of yearly diagnosed cancer cases. Cancer is a progressive and, in many cases, deadly disease of multicellular insurrection. The latest global cancer estimates from the International Agency for Research on Cancer (IARC) indicate that around 19.3 million cancer cases and 10.0 million cancer deaths occurred worldwide in 2020 [1]. Hepatocellular carcinoma (HCC) is the seventh most common malignancy in the world and is the third most frequent cause of cancer-related death [2]. A total of 840,000 new cases of liver cancer and 780,000 related deaths were recorded in 2018 [3]. According to previous studies, the 5-year relative survival rate of liver cancer patients was $18.4 \%$ (2009-2015) and $14.1 \%$ (2010-2014) in the USA and China $[4,5]$. According to comparative studies, results may differ between Asia-Pacific and Western patients and between different etiologies for the same therapeutic modalities [6, 7]. In a couple of regions, earlystage $\mathrm{HCC}$ benefits from potentially curative therapies $[8$, 9]. $25-70 \%$ of the patients are diagnosed with intermedi-

Correspondence to:

Yiping Mou, mouyiping@163.com

Xiujun Cai, caixiujunzju@163.com 
ate or advanced HCC, where treatment options are limited and prognosis is poor [10-12]. Approximately $75 \%$ of patients are in the Asia-Pacific region, and their cases are caused by hepatitis $B$ virus (HBV) and/or hepatitis $\mathrm{C}$ virus (HCV). More than $50 \%$ of cases of HCC occur in China, and an estimated 360,000 patients residing in east Asian countries, including China, Japan, Korea, and Taiwan, die from this disease each year [13-15]. Incidences of HCC are likely to increase over the next 10-20 years and to peak around 2030 [16]. Sorafenib and regorafenib could be of benefit, increase survival rates, and become the standard treatments for advanced HCC $[17,18]$. Another study showed that the promising systemic chemotherapeutic agent sorafenib only demonstrated relatively limited benefits rather than eradicating the microscopic residue and curing patients with advanced HCC. Therefore, sorafenib is beneficial in only around $30 \%$ of patients, and resistance often develops within 6 months [19]. The study by Jackson and his team [20] was consistent with evidence that the effectiveness of sorafenib on overall survival (OS) is dependent on patients' hepatitis status. There is an improved OS for patients negative for HBV but positive for HCV when treated by sorafenib. HCC related to infection with the HCV has become the fastest-rising cause of cancer-related death in the USA during the past 2 decades. The 5 -year survival rate for HCC has remained below 12\% [21]. According to a huge population-based study of individuals diagnosed with advanced HCC, the USA has not widely adopted sorafenib since FDA approval in 2007. Although sorafenib treatment is associated with a lower risk of mortality, this finding can be used to further the discussion surrounding appropriate adoption of sorafenib with clear and ongoing discussions of promoting cancer prevention [22, 23].

\section{Treatment Option for Patients with Advanced-Stage HCC}

Liver cancer (HCC) remains a major problem for health care systems around the world, and surgeons are facing the challenge of providing the most appropriate treatment for patients with HCC. The evaluation of prevention strategies, regular monitoring, and early diagnosis lead to good treatment options. Furthermore, immunotherapy drugs have brought new breakthroughs in the treatment of HCC $[24,25]$. The American Association for the Study of Liver Diseases (AASLD) published practice guidelines on the management of HCC in 2005. Since then, new information has emerged that requires the guidelines to be updated [26]. According to a publication by Bruix and colleagues [27], HCC patients have been defined (whether the nodules are 1 or $2 \mathrm{~cm}$ ), and effective treatment is available for tumors detected at an early stage. Several approaches are available at the intermediate-advance stage including radiofrequency ablation, transarterial chemoembolization, and radioembolization, and sorafenib remains the best option for advancedstage treatment [27]. However, a preliminary study by the SHARP-ORIENTAL Investigators Study Group showed that patients with advanced HCC, who received sorafenib treatment, had a median survival of nearly 10.7 months, as compared with 7.9 months [28]. Regorafenib is shown to provide survival benefits in HCC patients when added to sorafenib treatment, and 1-year survival is 33 and $44 \%$. Immune checkpoint inhibitors or oral multikinase inhibitors have been approved, notably lenvatinib (Lenvima; Eisai and Merck \& Co.), regorafenib (Stivarga; Bayer), cabozantinib (Cabometyx; Exelixis/Ipsen/Takeda), Ramucirumab (Cyramza; Eli Lilly), programmed cell death protein 1 (PD1) inhibitor nivolumab Opdivo (Opdivo; Bristol-Myers Squibb/Ono Pharmaceutical), PD1 inhibitor pembrolizumab (Keytruda; Merck \& Co.), the PD1 ligand 1 (PDL1) inhibitor, atezolizumab (Tecentriq; Roche/Genentech/Chugai), and the PDL1 inhibitor durvalumab (Imfinzi; AstraZeneca). The FDA granted atezolizumab plus bevacizumab breakthrough designation status as a first-line treatment for advanced or metastatic disease. Moreover, it is being evaluated in unresectable $\mathrm{HCC}$ and in patients with advanced or metastatic disease. Yet, the disease control rate was 35\% in the dose-escalation phase and $100 \%$ in the dose-expansion phase, which could have positively affected OS [29-31].

Although there have been significant advances in HCC treatment over the past decade, evidence is still lacking on therapeutics that can stop increasing incidence rates of HCC. Recently, the guidelines for the management of Chinese HCC patients were updated. The treatment of patients with HCC often necessitates multidisciplinary care (MDT). It includes the involvement of hepatologists, diagnostic and interventional radiologists, transplant surgeons, medical and surgical oncologists, and pathologists. The European Association for the Study of the Liver (EASL) and the American Association for the Study of Liver Diseases (AASLD) updated the clinical practical guidelines on the management of HCC in 2018, in line with typical standard management. Furthermore, the Asian Pacific Association for the Study of the Liver (APASL) HCC guidelines were published in 2010 and are the oldest of the major guidelines. The NCCN Guidelines 
for hepatobiliary cancers provide treatment recommendations for HCC $[16,32,33]$. China and other countries have established their guidelines for heterogeneity in the prevalence, phenotype, and treatment responses of HCC. The guidelines specific to Chinese patients were updated in 2019 based on an accumulation of new evidence [34, 35]. The study by Hatooka and colleagues [36] compared the evidence to determine whether hepatic arterial infusion chemotherapy (HAIC) or sorafenib is superior. In advanced-stage patients, they found favorable outcomes in patients who were treated with HAIC. Among responders, a better prognosis was expected with HAIC compared to sorafenib, while HAIC nonresponders had a poor prognosis at 6 months in previous studies, and 1and 2-year survival rates were higher. Therefore, it is necessary to identify HAIC nonresponders as early as possible. Also, the patients in the HAIC group remain alive for over 10 years [36].

\section{Comparison of Outcome of HAIC and Sorafenib in Patients with HCC Advanced Stage}

Recently, a few centers reported the outcomes of an interesting, randomized phase II trial comparing sorafenib alone (Sor) with sorafenib plus HAIC with cisplatin (SorCDDP) in advanced HCC. The median survivals in the Sor and SorCDDP arms were 8.7 and 10.6 months. The HR stratified by the allocation factors, including the presence/absence of portal vein tumor thrombosis (PVTT) and extrahepatic metastases $(95 \% \mathrm{CI})$, was 0.60 $(0.38-0.96)$ ( $p$ value 0.031$)$. The crude HR $(95 \% \mathrm{CI})$ was $0.68(0.44-1.049)(p=0.073)$. In brief, the administration of sorafenib before HAIC could lead to better clinical outcomes considering the sensitized and synergistic effect with cisplatin and the antiangiogenic activity of sorafenib. In a Korean study by Song and colleagues [39], on advanced-stage HCC and PVTT, 50 patients were treated with HAIC and 60 patients were treated with sorafenib. The median OS was significantly longer in the HAIC group than in the sorafenib group (7.1 vs. 5.5 months, $p$ $=0.011)$. The median time to progression was also significantly longer in the HAIC group than in the sorafenib group (3.3 vs. 2.1 months, $p=0.034$ ). So, HAIC shows more favorable responses compared to sorafenib [39].

Another randomized trial from Japan by Kudo and colleagues [40] (SILIUS) selected patients with advanced HCC not suitable for resection, local ablation, or transarterial chemoembolization. The outcome showed that HAIC plus sorafenib has shown promising tumor control and a manageable safety profile in patients in advanced stages and unresectable HCC. The median OS was similar for sorafenib plus HAIC and sorafenib (11.8 months [95\% CI: 9.1-14.5] vs. 11.5 months [8.2-14.8]; hazard ratio 1.009 [95\% CI: 0.743-1.371]; $p=0.955)$. Grade 3-4 adverse events (AEs) are more frequent in the sorafenib plus HAIC group than in the sorafenib group. Japanese Randomized HAIC outcomes are favorable and showed better responses for HCC patients exhibiting macroscopic vascular invasion when treated with HAIC rather than sorafenib [40]. Another Japanese review that reported treatment strategies for advanced HCC recommended sorafenib as the primary treatment for cases without macroscopic vascular invasion or Child-Pugh A and HAIC for those with macroscopic vascular invasion or ChildPugh A or B [41]. However, future research and further investigation is required for HAIC to become a standard treatment for HCC.

\section{HAIC, a Potential Therapeutic Option for HCC}

HAIC has been shown to be an effective treatment for advanced HCC in Asian countries, especially Japan [44]. According to the recent clinical practice guidelines for HCC in Japan (J-HCC 2013) [42], HAIC is one of the standard treatments for patients with advanced HCC. The clinical response was significant, and the responders had the best survival rates from 7 to $81 \%$, with a median survival time (MST) of approximately 6-15.9 months [43]. Theoretically, HAIC has a chemotherapeutic effect and a favorable toxicity profile in patients with advanced HCC. HAIC is one of the few remaining treatment options for advanced HCC patients in these predicaments because advanced HCC derives almost all of their blood supply from the hepatic artery. In HAIC, a highly concentrated chemotherapeutic agent is injected into the liver via the hepatic artery. The consequent concentration of the agent at the tumor site would be expected to increase antitumor effects [45]. Additionally, the liver derives about $70 \%$ of its blood supply from the portal vein. HAIC requires the insertion of a catheter into the hepatic artery. In single-administration HAIC, the catheter is inserted for a sole application of the chemotherapeutic agent each time. The methods for administering HAIC are dependent on certain agents. Epirubicin hydrochloride, mitomycin $\mathrm{C}$, cisplatin, and miriplatin are suitable for single administration, and the agents suitable for continuous administration include anthracycline-based agents, mitomycin C, and fluorouracil (5-FU) [43]. HAIC, on the 
other hand, is not recommended and should not be used as a standard of care. There is not enough convincing clinical evidence because large randomized studies of HAIC in advanced HCC patients have not been conducted. Recent studies continue to show high response rates, up to $52.6 \%$, and OS up to 36.8 months, using various combinations of modern agents. See Table 1.

\section{FAIT Therapeutic Option}

Fluorouracil arterial infusion plus interferon therapy (FAIT) was evaluated in patients with HCC with a high degree of vascular invasion associated with poor prognosis. FAIT showed beneficial effects for a combination therapy of interferon (IFN) and 5-FU, for advanced HCC with tumor thrombi in the major branches of the portal vein. The survival rates were from 30 to $40 \%$, with an MST of approximately 8.4-10.5 months [46-49]. A previous study by Obi and colleagues [46] evaluated FAIT in 116 patients; 19 patients (16\%) showed complete response and another 42 patients (36\%) showed partial response, with the response rate of $52.6 \%$, a median survival of 6.9 months, and a 1-year survival of $34 \%$, and prognosis improved when compared with a historical control [46]. Ota et al. [50] investigated FAIT in 55 patients with HCC and reported a response rate of $43.6 \%$, a median survival of 11.8 months, and a 1-year survival of $48.9 \%$ [50]. Recently, Yamashita et al. [47] reported that the response rate of FAIT was $24.6 \%$ (14 out of 57 patients), and the median survival was 10.5 months in patients with unresectable HCC, primarily due to high degrees of vascular invasion or multiple intrahepatic metastases [47]. Based on these findings (IFN and 5-FU), the authors evaluated the efficacy and safety of FAIT. FAIT may be a strategy of worth trying for advanced HCC with a high degree of vascular invasion.

\section{The Role of Low-Dose Cisplatin plus 5-FU for Treatment of Advanced and Recurrent HCC}

The efficacy of combination chemotherapy consisting of 5-FU and cisplatin (FP therapy) utilizing biochemical modulation for advanced HCC has been recently reported. Various regimens of HAIC have been tried individually or in combination, mainly using 5-FU or CDDP. 5-FU-based continuous infusion chemotherapy (FP or 5-FU/IFN) and CDDP monotherapy are typical regimens in Japan. However, there are few reports comparing the efficacy and safety of these regimens, and the basis of their selection is unclear [51-53]. One study by Nouso and colleagues [51] reported results of 476 patients with HCC who underwent HAIC with 5-FU and cisplatin (HAIC) compared with 1,466 patients who did not receive active therapy. The HAIC (HR, 0.48; 95\% CI: $0.41-0.56$; $p<$ $0.0001)$ was associated with better survival. The MSTs were 14.0 months (HAIC group) and 5.2 months (no therapy group), so survival was significantly higher in the HAIC group $(p<0.0001)$. Hazard ratio of HAIC in this propensity score-match was 0.60 (95\% CI: $0.49-0.73$; $p<$ 0.0001).

Most of the HCC treatment guidelines recommend chemotherapy for patients with Child-Pugh A/B disease who have portal vein invasion and/or $>3$ tumors. In cases of Child-Pugh A/B disease with $>3$ tumors, MST was 13.9 months (HAIC) and 3.7 months (without therapy), and the survival benefit of HAIC treatment was observed $(p<$ 0.0001). For other cases observed with Child-Pugh A/B disease with PVTT, MST was 7.9 months (HAIC) and 3.1 months (no therapy) [51]. Our findings and the previous study suggest that IFN with CDDP and 5-FU are currently used as HAIC regimens in Japan. The regimen containing IFN has not become widespread because there were not many clinical trials for these drugs. Low-dose FP is the most common regimen used for HAIC, and it has demonstrated a favorable outcome and can be important for achieving long-term survival in the future.

\section{Low-Dose Cisplatin Monotherapy Alone}

In Japan, CDDP has been available for intra-arterial infusion for the treatment of HCC since 2004. Recent studies reported that CDDP alone had an overall response rate of $33.8 \%$. The 1 -year survival rate was $67.5 \%$, and the 2 -year survival rate was $50.8 \%[54,55]$. HAIC has been used as a treatment option because of the efficacy and safety of HAIC using cisplatin for patients with earlystage HCC [56]. One Japanese study by Ishikawa and colleagues [57] studied the high efficacy and safety of using CDDP in patients with early-stage HCC. Some patients received radical local treatment with TACE and radiofrequency ablation, and other patients did not receive HAIC before radical local treatment. Outcomes showed that the cumulative survival rates at 1,3 , and 5 years in the nonHAIC group were $77.4,69.2$, and $55.3 \%$ and in the HAIC group were $97.4,87.0$, and $84.4 \%(p=0.023)$. The survival time was prolonged significantly in the HAIC group compared with the non-HAIC group. Based on these re- 
sults, treatments consisting of whole-liver HAIC with CDDP to all patients with early-stage HCC were appropriate for radical local treatment [57]. Also, anticancer agents are commonly used for the treatment of advanced HCC. Based on this finding, the cisplatin infusion significantly prolonged survival without increasing the incidence of AEs. These outcomes suggest that CDDP may play a leading role in determining that all-liver HAIC with CDDP is more beneficial as neoadjuvant or adjuvant therapy for HCC in the future.

\section{Potential Responders to FOLFOX Therapy}

Clinical activity of several regimens containing oxaliplatin in advanced and unresectable HCC has been demonstrated in phase II studies. FOLFOX (infusional fluorouracil, leucovorin, and oxaliplatin) administered to patients with advanced and unresectable HCC in Asia and the patients who were ineligible for curative resection or localize therapies could provide a survival benefit and greater efficacy compared with chemotherapy doxorubicin [58]. A Chinese study by Min and colleagues [59] compared the safety and efficacy of HAIC with FOLFOX versus TACE in patients with massive tumor and unresectable HCC. This study demonstrated encouraging outcomes for both efficacy and safety of HAIC with FOLFOX. The results of using HAIC with the FOLFOX group show higher and more significant partial responses and disease control rates than the TACE group rates. The results were 52.6 versus $9.8 \%, p<0.001 ; 83.8$ versus $52.5 \%, p=0.004$. The median survival rate in both HAIC and TACE groups was 5.87 and 3.6 months $(\mathrm{HR}=2.35$, $95 \% \mathrm{CI}=1.16-4.76, p=0.015)$. The number of patients who underwent surgical resection in the HAIC group was higher than the TACE group (10 vs. $3, p=0.033$ ). The result was not significant in either group. Also, the proportions of grade 3-4 AEs and serious AEs were lower in the HAIC group than in the TACE group (AEs: 13 vs. $27, p=0.007$; severe AEs: 6 vs. $15, p=0.044)$. Based on their findings, HAIC + FOLFOX yielded significant and better treatment responses and less serious toxicity than TACE and HAIC. FOLFOX might be a better choice for patients with unresectable HCC [59]. According to another study by Ning Lyu et al. [60], with a total of 55 enrolled patients with advanced HCC, almost 49 patients had cirrhosis caused by HBV infection. The aim of their study was to investigate the effect and safety of a HAIC + FOLFOX regimen in patients with advanced HCC. The responders had the best survival rates from
55.1 to $71.4 \%$, and the median survival rates were 6 and 12 months [60]. On the other hand, 2 studies SHARPORIENTAL Investigators Study Group and Barcelona Clinic Liver Cancer (BCLC) Group recommended sorafenib as the standard treatment for BCLC-C advanced-stage HCC [60]. Recently, the study by Ning Lyu et al. [60] treated 232 of 412 patients with sorafenib, and 180 patients were given HAIC + FOLFOX therapy. The median age range was $16-82$ years old. The results in both disease-free survival and OS in the HAIC + F group were significantly longer than those in the sorafenib group (disease-free survival 7.1 vs. 3.3 months; OS 14.5 vs. 7.0 months, $p<0.001$ ). This study found that HAIC + FOLFOX therapy may improve survival compared to sorafenib in patients with advanced HCC. Thus, HAIC $+\mathrm{F}$ is an alternative therapy for advanced HCC and is urgently required [61]. In brief, the combination of the HAIC with FOLFOX regimen in patients with advanced HCC has favorable survival rates, 6 and 12 months, high response rate, and acceptable toxicity and improves life quality; this strategy may provide a promising therapeutic option for advanced HCC.

\section{Prognostic Factors in Patients with HCC Receiving HAIC}

The safety and benefits of neoadjuvant HAIC in advanced HCC patients according to the Child-Pugh score and alpha-fetoprotein (AFP) level and the response to HAIC could certainly have survival benefits and may be a useful therapeutic option for patients with advanced HCC liver function and should be considered carefully when dealing with HAIC [62-64]. The radiological response to therapy was assessed by modified response evaluation criteria in both solid tumors and classified as complete response (CR), partial response (PR), stable disease (SD), or progressive disease (PD). Early response was defined as the achievement of CR or PR, and disease control was defined as the achievement of CR, PR, or SD. To evaluate biologic tumor response, the alterations in AFP were analyzed based on primer AFP value after treatment [64], according to Miyaki et al. [63]. The early response results and outcomes of HAIC and MST with early imaging response are MST in PR, 20.6; SD, 11.4; and PD, 5.0 months $(p<0.0001)$. The MST was significantly different in patients with AFP and DCP of $\leq 1$ or $>1$. Worse MST of 6.5 months was seen in patients with an AFP ratio of $>1$ and a DCP ratio of $>1$. Yet, decreases in both AFP and DCP in early-stage therapy with HAIC were significant, 
Table 1. Different studies of HAIC with systemic therapy for liver cancer [37, 38, 47, 51, 57, 59, 61, 63]

\begin{tabular}{|c|c|c|c|c|c|c|c|}
\hline Study & $\begin{array}{l}\text { Study } \\
\text { design }\end{array}$ & Year & $\begin{array}{l}\text { Patients, } \\
n\end{array}$ & $\begin{array}{l}\text { Median } \\
\text { age, years }\end{array}$ & Treatment & $\mathrm{RR}, \%$ & $\begin{array}{l}\text { OS, } \\
\text { months }\end{array}$ \\
\hline Ikeda et al. [37] & $\begin{array}{l}\text { Multicenter } \\
\text { RCT }\end{array}$ & 2016 & 65 & 64 & Sorafenib + HAIC (cisplatin) & 21.7 & 10.6 \\
\hline Song et al. [38] & $\begin{array}{l}\text { Multicenter } \\
\text { RCT }\end{array}$ & 2014 & 50 & 55.1 & HAIC and sorafenib, cisplatin $+5-\mathrm{FU}$ & 24.0 & 7.1 \\
\hline Yamashita et al. [47] & $\begin{array}{l}\text { Single-center } \\
\text { RCT }\end{array}$ & 2011 & 57 & 65 & $\begin{array}{l}\text { Hepatic arterial infusion of fluorour } \\
\text { acil plus cisplatin }\end{array}$ & 45.6 & 10.5 \\
\hline Nouso et al. [51] & $\begin{array}{l}\text { Multicenter } \\
\text { RCT }\end{array}$ & 2013 & 476 & - & Cisplatin $+5-\mathrm{FU}$ & 40.5 & 14.0 \\
\hline Ishikawa et al. [57] & $\begin{array}{l}\text { Single-center } \\
\text { RCT }\end{array}$ & 2014 & 79 & 67.8 & HAI with DDP-H & - & 36.8 \\
\hline Min et al. [59] & $\begin{array}{l}\text { Single-center } \\
\text { prospective }\end{array}$ & 2017 & 38 & 71.1 & HAIC (mFOLFOX) & 52.6 & 5.87 \\
\hline
\end{tabular}

HAIC, hepatic artery infusion chemotherapy; 5-FU, 5-fluorouracil; RCT, randomized controlled trial; RR, response rate; OS, overall survival; IFN, interferon; DDP-H, cis-diamminedichloroplatinum; HAI, hepatic arterial infusion.

and independent prognostic factors associated with OS in all patients [63]. See Table 1.

Another study by Niizeki and colleagues [65] reported the serum vascular endothelial growth factor levels and the therapeutic effect of HAIC with advanced HCC patients. Various chemotherapeutic regimens are used for HAIC, with the combination of cisplatin (CDDP) and 5 -FU being one of the most common and standard regimens, and the serum vascular endothelial growth factor level was an important predictive factor of the therapeutic effect and survival in patients with advanced HCC undergoing HAIC [65]. Ando et al. [62] first reported that repeated HAIC using low-dose CDDP and 5-FU was useful for patients with advanced HCC and tumor thrombus in the portal vein $[62,66]$. A previous study by Ota and colleagues [50] reported the beneficial effects of combination therapy of IFN- $\alpha / 5-F U$ which is promising for advanced HCC with tumor [50]. In addition, a study by Tajiri and colleagues [67] reported on 26 patients with advanced HCC who were treated by HAIC using arterial cisplatin plus continuous 5-FU. The neutrophil/lymphocyte ratio (NLR) was assessed to determine its effectiveness as a prognostic indicator of HAIC. The response rate was $42.3 \%$ and was independent of liver function. Response rate was significantly lower in patients with an NLR of 4 . Therefore, the significance of NLR in predicting the effects of HAIC-FP remains uncertain. So, inves- tigations are required [67]. Finally, and briefly, inflammation plays a critical role in tumors, and the oncogenic change induces an inflammatory microenvironment that promotes the development of tumors.

\section{Conclusion}

Our conclusion is that HAIC is a promising and acceptable approach for the management of advanced HCC with tolerable toxicities. Furthermore, various novel combinations of chemotherapeutic regimens have been recognized to be useful as standard treatments for advanced HCC patients. The previous randomized trials of combination therapies showed better benefits in patients who were treated with a combination of cisplatin $+5-\mathrm{FU}$, HAIC + sorafenib, FOLFOX, and INF + 5-FU. On the basis of these findings, we hope future studies will demonstrate more attractive results and publish more trials. Recently, HAIC has been researched widely. Thus, further improvements in the treatment outcomes of patients with advanced HCC are expected in the near future.

\section{Conflict of Interest Statement}

The authors declare that they have no conflicts of interest. 


\section{Funding Sources}

The authors declare that they have no funding.

\section{Author Contributions}

Maher Hendi and Xiujun Cai interpreted data and wrote the manuscript. Yiping Mou, Jiemin Lv, and Bin Zhang revised the manuscript. Maher Hendi and Yiping Mou designed the work. All authors discussed the results and contributed to the final version of this manuscript.

\section{References}

1 International Agency dor Research on Cancer. Available from: https://www.iarc.fr/ news-events/latest-global-cancer-data-cancer-burden-rises-to-19-3- illion-new-casesand-10-0-million-cancer-deaths-in-2020/.

2 Lin L, Yan L, Liu Y, Qu C, Ni J, Li H. The Burden and trends of primary liver cancer caused by specific etiologies from 1990 to 2017 at the global, regional, national, age, and sex level results from the global burden of disease study 2017. Liver Cancer. 2020 Sep;9(5):56382.

3 Bray F, Ferlay J, Soerjomataram I, Siegel RL, Torre LA, Jemal A. Global cancer statistics 2018: GLOBOCAN estimates of incidence and mortality worldwide for 36 cancers in 185 countries. CA Cancer J Clin. 2018 Nov;68(6): 394-424.

4 Allemani C, Matsuda T, Di Carlo V, Harewood R, Matz M, Nikšić M, et al. Global surveillance of trends in cancer survival 2000-14 (CONCORD-3): analysis of individual records for 37,513,025 patients diagnosed with one of 18 cancers from 322 population-based registries in 71 countries. Lancet. 2018 Mar; 391(10125):1023-75.

5 National Cancer Institute. Surveillance, Epidemiology, and End Results Program. Available from: https://seer.cancer.gov/statfacts/ html/livibd.html. Accessed 2019 Sep.

6 Franssen B, Alshebeeb K, Tabrizian P, Marti J, Pierobon ES, Lubezky N, et al. Differences in surgical outcomes between hepatitis B- and hepatitis C-related hepatocellular carcinoma: a retrospective analysis of a single North American center. Ann Surg. 2014;260:650-8; discussion 656-658.

7 Park JW, Chen M, Colombo M, Roberts LR, Schwartz M, Chen PJ, et al. Global patterns of hepatocellular carcinoma management from diagnosis to death: the BRIDGE Study. Liver Int. 2015;35:2155-66.

8 Poon D, Anderson BO, Chen LT, Tanaka K, Lau WY, Van Cutsem E, et al. Management of hepatocellular carcinoma in Asia: consensus statement from the Asian oncology summit 2009. Lancet Oncol. 2009;10:1111-8.

9 Llovet JM, Fuster J, Bruix J. The Barcelona approach: diagnosis, staging, and treatment of hepatocellular carcinoma. Liver Transpl. 2004;10:S115-20.

10 Cheng AL, Kang YK, Chen Z, Tsao CJ, Qin $S$, Kim JS, et al. Efficacy and safety of sorafenib in patients in the Asia-Pacific re- gion with advanced hepatocellular carcinoma: a phase III randomised, double-blind, placebo-controlled trial. Lancet Oncol. 2009; 10:25-34.

11 Chow PK, Tai BC, Tan CK, Machin D, Win $\mathrm{KM}$, Johnson PJ, et al. High-dose tamoxifen in the treatment of inoperable hepatocellular carcinoma: a multicenter randomized controlled trial. Hepatology. 2002;36:1221-6.

12 Pawarode A, Tangkijvanich P, Voravud N. Outcomes of primary hepatocellular carcinoma treatment: an 8-year experience with 368 patients in Thailand. J Gastroenterol Hepatol. 2000;15:860-4.

13 McGlynn KA, Tsao L, Hsing AW, Devesa SS, Fraumeni JF Jr. International trends and patterns of primary liver cancer. Int J Cancer. 2001;94:290-6

14 Kirk GD, Lesi OA, Mendy M, Akano AO, Sam $\mathrm{O}$, Goedert JJ, et al. The gambia liver cancer study: infection with hepatitis B and C and the risk of hepatocellular carcinoma in West Africa. Hepatology. 2004;39:211-9.

15 Parkin DM. Global cancer statistics in the year 2000. Lancet Oncol. 2001;2:533-43.

16 Omata M, Cheng A, Norihiro K, Masatoshi K, Jeong $\mathrm{M}$, Jidong J, et al. Asia-Pacific clinical practice guidelines on the management of hepatocellular carcinoma: a 2017 update. Hepatol Int. 2017 Jul;11(4):317-70.

17 Bruix J, Reig M, Sherman M. Evidence-based diagnosis, staging, and treatment of patients with hepatocellular carcinoma. Gastroenterology. 2016;150:835-53.

18 Forner A, Reig M, Bruix J. Hepatocellular carcinoma. Lancet. 2018;391:1301-14.

19 Chen J, Zhao J, Liu J, Ying H, Han Y, Zhou S, et al. Potential molecular, cellular and microenvironmental mechanism of sorafenib resistance in hepatocellular carcinoma. Cancer Letter. 2015;367(1):1-11.

20 Jackson R, Psarelli EE, Berhane S, Khan H, Johnson P. Impact of viral status on survival in patients receiving sorafenib for advanced hepatocellular cancer: a meta-analysis of randomized phase III trials. J Clin Oncol. 2017; 35:622-8.

21 El-Serag HB. Hepatocellular carcinoma. N Engl J Med. 2011;365:1118-27.

22 Parsons HM, Chu Q, Karlitz JJ, Stevens JL, Harlan LC. Adoption of sorafenib for the treatment of advanced-stage hepatocellular carcinoma in oncology practices in the United States. Liver Cancer. 2017;6:216-26.
23 Park JW, Chen M, Colombo M, Roberts LR, Schwartz M. Global patterns of hepatocellular carcinoma management from diagnosis to death: the BRIDGE Study. Liver Int. 2015;35: 2155-66.

24 Petrick JL, Florio AA, Znaor A, Ruggieri D, Laversanne M, Alvarez CS, et al. International trends in hepatocellular carcinoma incidence, 1978-2012. Int J Cancer. 2019;147: 317-30.

25 Bertuccio P, Turati F, Carioli G, Rodriguez T, La Vecchia C, Malvezzi M, et al. Global trends and predictions in hepatocellular carcinoma mortality. J Hepatol. 2017;67:302-9.

26 Bruix J, Morris S. Management of hepatocellular carcinoma: an update. Hepatology. 2011;53:1020-2.

27 Bruix J, Gores G, Mazzaferro V. Hepatocellular carcinoma: clinical frontiers and perspectives. Gut. 2014;63:844-55.

28 Llovet J, Sergio R, Vincenzo M, Philip H, Edward G, Frédéric Blanc J. Sorafenib in advanced hepatocellular carcinoma. $\mathrm{N}$ Engl J Med. 2008;359:378-90.

29 Dawkins J, Webster RM. The hepatocellular carcinoma market. Nat Rev Drug Discov. 2019 Jan;18(1):13-4.

30 Bruix J, Qin S, Merle P, Granito A, Huang YH, Bodoky G, et al. Regorafenib for patients with hepatocellular carcinoma who progressed on sorafenib treatment (RESORCE): a randomised, double-blind, placebo-controlled, phase 3 trial. Lancet. 2016;389:56-66.

31 El-Khoueiry AB, Sangro B, Yau T, Crocenzi TS, Kudo M, Hsu C, et al. Nivolumab in patients with advanced hepatocellular carcinoma (CheckMate 040): an open-label, noncomparative, phase $1 / 2$ dose escalation and expansion trial. Lancet. 2017;389:2492-502.

32 European Association for the Study of the Liver. EASL clinical practice guidelines: management of hepatocellular carcinoma. J Hepatol 2018; 69:182-236.

33 Marrero JA, Kulik LM, Sirlin CB, Zhu AX, Finn RS, Abecassis MM, et al. Diagnosis, staging, and management of hepatocellular carcinoma: 2018 practice guidance by the American Association for the Study of liver diseases. Hepatology. 2018;68:723-50.

34 Zhou J, Sun HC, Wang Z, Cong WM, Wang $\mathrm{JH}$, Zeng MS, et al. Guidelines for diagnosis and treatment of primary liver cancer in China (2017 Edition). Liver Cancer. 2018;7:23560 . 
35 Department of Medical Administration; National Health and Health Commission of the People's Republic of China. Guidelines for diagnosis and treatment of primary liver cancer in China (2019. Edition). Chin J Hepatol. 2020;28(2):112-28.

36 Hatooka M, Kawaoka T, Aikata H, Inagaki Y, Morio K, Nakahara T, et al. Hepatic arterial infusion chemotherapy followed by sorafenib in patients with advanced hepatocellular carcinoma (HICS 55): an open label, non-comparative, phase II trial. BMC Cancer. 2018; 18(1):633.

37 Ikeda M, Shimizu S, Sato T, Morimoto M, Kojima Y, Inaba Y, et al. Sorafenib plus hepatic arterial infusion chemotherapy with cisplatin versus sorafenib for advanced hepatocellular carcinoma: randomized phase II trial. Ann Oncol. 2016;27:2090-6.

38 Ikeda M, Shimizu S, Sato T, Morimoto M, Kojima Y, Inaba Y, et al. Reply to the Letter to the editor 'Sorafenib plus hepatic arterial infusion chemotherapy with cisplatin versus Sorafenib for advanced hepatocellular carcinoma: randomized phase II trial' by Fornaro et al. Ann Oncol. 2017;28:903-4.

39 Song DS, Song MJ, Bae SH, Chung WJ, Jang JY, Kim YS, et al. A comparative study between sorafenib and hepatic arterial infusion chemotherapy for advanced hepatocellular carcinoma with portal vein tumor thrombosis. J Gastroenterol. 2015 Apr;50(4):445-54.

40 Kudo M, Ueshima K, Yokosuka O, Ogasawara S, Obi S, Izumi N, et al. Sorafenib plus low-dose cisplatin and fluorouracil hepatic arterial infusion chemotherapy versus sorafenib alone in patients with advanced hepatocellular carcinoma (SILIUS): a randomised, open label, phase 3 trial. Lancet Gastroenterol Hepatol. 2018;3:424-32.

41 Saeki I, Yamasaki T, Maeda M, Hisanaga T, Iwamoto T, Fujisawa K, et al. Treatment strategies for advanced hepatocellular carcinoma: sorafenib vs. hepatic arterial infusion chemotherapy. World J Hepatol. 2018;10(9):571-84.

42 Japan Society of Hepatology Clinical practice guidelines for hepatocellular carcinoma $(2013$ version). Kanehara, Tokyo, Japan, 2013. (in Japanese) An English translation of the guidelines is available at the following. Available from: http://www.jsh.or.jp/English/guidelines_en/Guidelines_for_hepatocellular_carcinoma_2013.

43 Obi S, Sato S, Kawai T. Current status of hepatic arterial infusion chemotherapy. Liver Cancer. 2015;4:188-99.

44 Ueshima K, Kudo M, Takita M, Nagai T, Tatsumi $C$, Ueda $T$, et al. Hepatic arterial infusion chemotherapy using low-dose 5-fluorouracil and cisplatin for advanced hepatocellular carcinoma. Oncology. 2010;78(Suppl 1):148-53.

45 Okada S. Chemotherapy in hepatocellular carcinoma. Hepatogastroenterology. 1998; 45(Suppl 3):1259-63.

46 Obi S, Yoshida H, Toune R, Unuma T, Kanda $\mathrm{M}$, Sato S, et al. Combination Therapy of intraarterial 5-fluorouracil and systemic inter- feron-alpha for advanced hepatocellular carcinoma with portal venous invasion. Cancer. 2006; 106:1990.

47 Yamashita T, Arai K, Sunagozaka H, Ueda T, Terashima T, Yamashita T, et al. Randomized, phase II study comparing interferon combined with hepatic arterial infusion of fluorouracil plus cisplatin and fluorouracil alone in patients with advanced hepatocellular carcinoma. Oncology. 2011;81(5-6):28190.

48 Monden M, Sakon M, Sakata Y, Ueda Y, Hashimura E; FAIT Research Group. 5-fluorouracil arterial infusion + interferon therapy for highly advanced hepatocellular carcinoma: a multicenter, randomized, phase II study. Hepatol Res. 2012;42:150-65.

49 Ikeda M, Okusaka T, Furuse J, Mitsunaga S, Ueno H, Yamaura H. A multi-institutional phase II trial of hepatic arterial infusion chemotherapy with cisplatin for advanced hepatocellular carcinoma with portal vein tumor thrombosis. Cancer Chemother Pharmacol. 2013;72:463-70.

50 Ota H, Nagano H, Sakon M, Eguchi H, Kondo M, Yamamoto T, et al. Treatment of hepatocellular carcinoma with major portal vein thrombosis by combined therapy with subcutaneous interferon- $\alpha$ and intra-arterial 5-fluorouracil; role of type 1 interferon receptor expression. Br J Cancer. 2005; 93(5):557-64.

51 Nouso K, Miyahara K, Uchida D, Kuwaki K, Izumi $\mathrm{N}$, Omata $\mathrm{M}$, et al. Effect of hepatic arterial infusion chemotherapy of 5-fluorouracil and cisplatin for advanced hepatocellular carcinoma in the Nationwide Survey of Primary Liver Cancer in Japan. Br J Cancer. 2013;109(7):1904-7.

52 Kawaoka T, Aikata H, Kobayashi T, Uchikawa S, Ohya K, Kodama K, et al. Comparison of hepatic arterial infusion chemotherapy between 5-fluorouracil-based continuous infusion chemotherapy and low-dose cisplatin monotherapy for advanced hepatocellular carcinoma. Hepatol Res. 2018;48:1118-30.

53 Ueshima K, Kudo M, Tanaka M, Kumada T, Chung H, Hagiwara S, et al. Phase I/II Study of sorafenib in combination with hepatic arterial infusion chemotherapy using low-dose cisplatin and 5-fluorouracil. Liver Cancer. 2015;4:263-73.

54 Yoshikawa $\mathrm{M}$, Ono $\mathrm{N}$, Yodono $\mathrm{H}$, Ichida $\mathrm{T}$, Nakamura H, et al. Phase II study of hepatic arterial infusion of a fine-powder formulation of cisplatin for advanced hepatocellular carcinoma. Hepatol Res. 2008;38:474-83.

55 Kondo M, Morimoto M, Numata K, Nozaki A, Tanaka K. Hepatic arterial infusion therapy with a fine powder formulation of cisplatin for advanced hepatocellular carcinoma with portal vein tumor thrombosis. Jpn J Clin Oncol. 2011;41(1):69-75.

56 Kim BK, Park JY, Choi HJ, Kim DY, Ahn SH, Kim JK, et al. Long-term clinical outcomes of hepatic arterial infusion chemotherapy with cisplatin with or without 5-fluorouracil in lo- cally advanced hepatocellular carcinoma. J Cancer Res Clin Oncol. 2011;137:659-67.

57 Ishikawa T, Kubota T, Abe S, Watanabe Y, Sugano $\mathrm{T}$, Inoue $\mathrm{R}$, et al. Hepatic arterial infusion chemotherapy with cisplatin before radical local treatment of early hepatocellular carcinoma (JIS score 0/1) improves survival. Ann Oncol. 2014;25:1379-84.

58 Qin S, Bai Y, Lim HY, Thongprasert S, Chao $\mathrm{Y}$, Fan J, et al. Randomized, multicenter, open-label study of oxaliplatin plus fluorouracil/leucovorin versus doxorubicin as palliative chemotherapy in patients with advanced hepatocellular carcinoma from Asia. J Clin Oncol. 2013;31(28):3501-8.

59 Min HE, Le Y, Li QJ, Yu ZS, Li SH, Wei W, et al. Hepatic artery infusion chemotherapy using mFOLFOX versus transarterial chemoembolization for massive unresectable hepatocellular carcinoma:a prospective non-randomized study. Chin J Cancer. 2017;36(1):83.

60 Ning L, Lin Y, Kong Y, Zhang Z, Liu L, Zheng

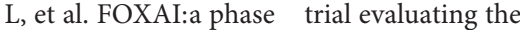
efficacy and safety of hepatic arterial infusion of oxaliplatin plus fluorouracil/leucovorin for advanced hepatocellular carcinoma. Gut. 2018;67(2):391-5.

61 Lyu N, Kong Y, Mu L, Lin Y, Li J, Liu Y, et al. Hepatic arterial infusion of oxaliplatin plus fluorouracil/leucovorin vs. sorafenib for advanced hepatocellular carcinoma. J Hepatol. 2018;69(1):60-9.

62 Ando E, Tanaka M, Yamashita F, Kuromatsu R, Yutani S, Fukumori K, et al. Hepatic arterial infusion chemotherapy for advanced hepatocellular carcinoma with portal vein tumor thrombosis:analysis of 48 cases. Cancer. 2002;95(3):588-95.

63 Miaki D, Aikata H, Honda Y, Naeshiro N, Nakahara T, Tanaka M, et al. Hepatic arterial infusion chemotherapy for advanced hepatocellular carcinoma according to Child-Pugh classification. J Gastroenterol Hepatol. 2012; 27(12):1850-7

64 Lee BH, Lee DS, Cho CW, Yun SS. Role and limitation of neoadjuvant hepatic arterial infusion chemotherapy in advanced hepatocelluar carcinoma patients with Child-Pugh class A. World J Surg Oncol. 2019;17:143.

65 Niizeki T, Sumi S, Torimura T, Kurogi J, Kuromatsu $\mathrm{R}$, Iwamoto $\mathrm{H}$, et al. Serum vascular endothelial growth factor as a predictor of response and survival in patients with advanced hepatocellular carcinoma undergoing hepatic arterial infusion chemotherapy. J Gastroenterol. 2012;47(6):686-95.

66 Ando E, Yamashita F, Tanaka M, Tanikawa K. A novel chemotherapy for advanced hepatocellular carcinoma with tumor thrombosis of the main trunk of the portal vein. Cancer. 1997;79:1890-6.

67 Tajiri K, Kawai K, Minemura M, Yasumura S, Hosokawa A, Kawabe H, et al. Neutrophil/ lymphocyte ratio as a prognostic indicator of hepatic arterial infusion chemotherapy with arterial cisplatin plus continuous 5-fluorouracil. Hepatol Res. 2015;45(7):755-63. 